\title{
Medicine recalls in Saudi Arabia: a retrospective review of drug alerts (January 2010-January 2019)
}

\author{
Bushra T. AlQuadeib* (D), Iman M. Alfagih, Ameera H. Alnahdi, Shadia M. Alharbi and Rawan A. Al-ahmari
}

\begin{abstract}
Background: A new incidence of a substandard medicinal product is discovered weekly as stated by the World Health Organization (WHO) around the world. After discovering an incidence of a substandard medicinal product, a drug recall announced to remove the affected medicinal product from the market. Drug recalls in SaudiArabia (SA) are made by the Saudi Food and Drug Authority (SFDA) or the distributors of the drug when certain criteria are met. A retrospective study of drug recalls in SA was carried out. Data were collected through a website search of the SFDA for drug recalls since January 2010-January 2019.

Results: SFDA recalled 84 substandard medicinal products which related to 47 alert letters and 52 drugs. The number of drug recall reported by SFDA increased six-folds in 2018 than in 2010. The major frequent therapeutic class of drug recall was the antihypertensive drugs followed by the antibiotic drugs ( $27.7 \%$ and $10.8 \%$, respectively). The majority of the recalls were tablets followed by parenteral dosage forms ( $58 \%$ and $25 \%$, respectively). The major reasons for the drug recalls were due to contamination (32.14\%) followed by non-compliance with manufacturer's specifications (20.48\%). Two manufacturers were accounted for $34.52 \%$ of all recalled drugs.
\end{abstract}

Conclusion: Substandard medicinal products are considered as a serious problem in SA. Contamination was the main cause of drug recalls, which requires root investigation of causes and for strict protective tools to be applied by drug manufacturers.

Keywords: Drug recall, Substandard, Falsified, Saudi FDA, Review

\section{Background}

Recently, an increase in records of medicinal recalls (prescribed and/or over the counter drugs) was reported worldwide. When a medicinal product is removed from the market, it is called drug recall. It may be occurred due to contaminations with other ingredients or the presence of adverse effects associated with medicine administration [1-12]. A drug recall is a significant tool that can decrease the morbidity and mortality due to problems in medicinal product manufacturing and distribution. This was evident in 2005 in the outbreak of bloodstream infections (total no. cases, 80 with no

\footnotetext{
* Correspondence: bquadeib@ksu.edu.sa

Department of Pharmaceutics, College of Pharmacy, King Saud University, P.O. Box 84428, Riyadh 11671, Saudi Arabia
}

deaths) caused by $P$. fluorescens contaminated heparinized saline intravenous flush syringes [13].

In 2017, the World Health Organization (WHO) agreed on definitions proposed to specify what may be classified as defective medicinal products. WHO indicates that medicinal products are to be denoted as substandard medical products "these are authorized medical products that fail to meet either their quality standards or specifications, or both," whereas falsified medical products are "medicinal products that deliberately/ fraudulently misrepresent their identity, composition or source" [14].

Although all these definitions may not incarcerate the wide range of substandard medicinal products including those that were expired, inactive, or improperly stored/

\section{Springer Open}

(c) The Author(s). 2020 Open Access This article is licensed under a Creative Commons Attribution 4.0 International License, which permits use, sharing, adaptation, distribution and reproduction in any medium or format, as long as you give appropriate credit to the original author(s) and the source, provide a link to the Creative Commons licence, and indicate if changes were made. The images or other third party material in this article are included in the article's Creative Commons licence, unless indicated otherwise in a credit line to the material. If material is not included in the article's Creative Commons licence and your intended use is not permitted by statutory regulation or exceeds the permitted use, you will need to obtain permission directly from the copyright holder. To view a copy of this licence, visit http://creativecommons.org/licenses/by/4.0/. 
transported [2]. These arguments in definitions further obscure global management and local regulation of counterfeits and point to the need for an internationally accepted definition of what constitutes a "counterfeit" medicine [2, 15-17].

In Saudi Arabia, the Saudi Food and Drug Authority (SFDA) is the responsible firm of protecting the public health from the danger of medicinal product defects. The drug sector at SFDA is the department responsible for receiving and evaluating complaints about medicinal products. The SFDA was founded under the Council of Ministers in 2003 and launched in 2009, as an independent body corporate. The main objective for the SFDA is to ensure the safety of food and drug for humans and animals, as well as electronic medical instruments $[18,19]$.

The SFDA defines a drug with a quality defect "any drug with faulty manufacture, product deterioration, detection of falsification, non-compliance with the marketing authorization or product specification file, or any other serious quality problem" [20].

Drug alerts are released by the SDFA to the manufacturer, distributor, healthcare professional, and public, when a defective medicine is reported to threat patients' safety. SFDA has several tools for delivering drug alerts to the public and to healthcare providers. All recalls are made available to the public via Saudi Drug Bulletin on SFDA's website or posted on drug alerts and news page on SFDA website. Since 2017, there is an integrated site for drug recall, which opened an important communication tool with the public, healthcare providers, pharmacies, drug manufacturers, and distributors [19, 21-24]. When drug recalls are announced, public notification occurred to stop using this product and discard any unused amount and additionally, counseling the public what to do and the best alternative choice to maintain patient safety with an efficient safe drug.

In February 2019, SFDA inaugurated the electronic tracking system for pharmaceutical monitoring. Through this system, the authority aims to track pharmaceutical products electronically at all stages of production and consumption ensuring the availability of medicines and their safety, in cooperation with government and private hospitals, clinics and health centers, government and commercial warehouses dedicated to the import and storage of medicines, community pharmacies, and the final consumer of the drug. This monitoring system contributes to the reduction of the circulation of products with quality defect, the provision of medicines, and their location, in addition to achieving drug safety by stopping the circulation of withdrawn or suspended drugs [24].

This is the first review to study the drug recall in SA and initiate a collection of recall medication letters from the SFDA website. The aims of this study were to analyze and characterize drug alerts reported by the
SFDA from Jan 2010 to Jan 2019, excluding herbal and/ or cosmetic drugs. It also discusses the number of the drug recall per year, the major therapeutic drug recall class, the most pharmaceutical dosage form recalls, and the reasons for recalls.

\section{Methods}

Drug alerts about recalled drugs were collected from the official SFDA website through the section assigned for drug news and alerts, and the section assigned for drug recall which was launched in 2017. The search includes all drug alerts reported between Jan 2010 and Jan 2019 excluding herbal and cosmetics recalls.

The following information was extracted from drug alerts: the name of the medicinal product, the name of the active ingredient(s), dosage form, strength, manufacturer; batch number affected; the date and recall reason; and the subsequent action taken by SFDA.

The drug recalls collected were categorized into one of the following groups: defective medicinal products, falsified medicinal products, or adverse reactions. Defective medicinal products were then reclassified depending on the type of the defect into the following: contamination, non-compliance with manufacturer's specification, packaging defect, failure to demonstrate bioequivalence, and problems related to the active ingredients. The medicinal products were classified as falsified only when it was stated in the drug alert that the product was considered by the SFDA as falsified medicinal products. The authors were unable to determine if the defect was nonintentional or intentional because no data was available to support their statement.

Moreover, recalled medicinal products were classified by the WHO Anatomical Therapeutic Chemical (ATC) Classification System. In this classification, the first category classifies medicinal products according to the main therapeutic class. This was carried out to underline the most frequent therapeutic groups affected by these recalls [25]. Also, the most common manufacturers of recalled medicinal products were identified.

\section{Results}

Recall letters were collected from SFDA website, and from it, the identification of the recall drug, name, and reason for the recall was listed in Table 1 . SFDA issued a total of 84 drug recalls during the period of the study January 2010-January 2019, consisting of 47 letters of drug recalls which include 52 drug generic names, and five of these drug recalls $(6 \%)$ were not registered in SFDA (Table 1). Most of these recalls were reported for one product, although some were reported for up to six drug products.

Unfortunately, during the period of the study, drug recalls increased from nine drug recalls in 2010 (10.71\%) 
Table 1 Drug recalls data (January 2010 - January 2019)

\begin{tabular}{|c|c|c|c|c|}
\hline $\begin{array}{l}\text { No. of } \\
\text { recall } \\
\text { letters }\end{array}$ & $\begin{array}{l}\text { No. of } \\
\text { recall } \\
\text { drugs }\end{array}$ & Trade name & Reference of recall (web link) & Reason of recall \\
\hline \multirow[t]{4}{*}{1} & 1 & Sibutral $^{\circledast}$ & $\begin{array}{l}\text { https://www.sfda.gov.sa/ar/drug/news/ } \\
\text { pages/699-ar-7-3.aspx }\end{array}$ & Lead to serious side effects such as cardiovascular disease \\
\hline & 2 & Reductil ${ }^{\oplus}$ & $\begin{array}{l}\text { https://www.sfda.gov.sa/ar/drug/news/ } \\
\text { pages/699-ar-7-3.aspx }\end{array}$ & Lead to serious side effects such as cardiovascular disease \\
\hline & 3 & Avandary $\left.\right|^{\circledast}$ & $\begin{array}{l}\text { https://www.sfda.gov.sa/ar/drug/news/ } \\
\text { Pages/703-ar-15-3.aspx }\end{array}$ & Lead to serious side effects such as cardiovascular disease \\
\hline & 4 & Avandamet ${ }^{\oplus}$ & $\begin{array}{l}\text { https://www.sfda.gov.sa/ar/drug/news/ } \\
\text { Pages/703-ar-15-3.aspx }\end{array}$ & Lead to serious side effects such as cardiovascular disease \\
\hline \multirow[t]{3}{*}{2} & 5 & Avandia ${ }^{\oplus}$ & $\begin{array}{l}\text { https://www.sfda.gov.sa/ar/drug/news/ } \\
\text { Pages/703-ar-15-3.aspx }\end{array}$ & Lead to serious side effects such as cardiovascular disease \\
\hline & 6 & Serevent ${ }^{\oplus}$ & $\begin{array}{l}\text { https://www.sfda.gov.sa/ar/drug/news/ } \\
\text { Pages/911-ar-6-11.aspx }\end{array}$ & $\begin{array}{l}\text { Increased risk of asthma complications requiring } \\
\text { hospitalization in children and adults }\end{array}$ \\
\hline & 7 & Sere vent ${ }^{\oplus}$ & $\begin{array}{l}\text { https://www.sfda.gov.sa/ar/drug/news/ } \\
\text { Pages/911-ar-6-11.aspx }\end{array}$ & $\begin{array}{l}\text { Increased risk of asthma complications requiring } \\
\text { hospitalization in children and adults }\end{array}$ \\
\hline \multirow[t]{2}{*}{3} & 8 & $\mathrm{Oxis}^{\oplus}$ & $\begin{array}{l}\text { https://www.sfda.gov.sa/ar/drug/news/ } \\
\text { Pages/911-ar-6-11.aspx }\end{array}$ & $\begin{array}{l}\text { Increased risk of asthma complications requiring } \\
\text { hospitalization in children and adults }\end{array}$ \\
\hline & 9 & Foradil ${ }^{\oplus}$ & $\begin{array}{l}\text { https://www.sfda.gov.sa/ar/drug/news/ } \\
\text { Pages/911-ar-6-11.aspx }\end{array}$ & $\begin{array}{l}\text { Increased risk of asthma complications requiring } \\
\text { hospitalization in children and adults. }\end{array}$ \\
\hline 4 & 10 & Augmentin ${ }^{\oplus}$ & $\begin{array}{l}\text { https://www.sfda.gov.sa/ar/drug/news/ } \\
\text { Pages/777-31-10.aspx }\end{array}$ & Free from active substances \\
\hline 5 & 11 & $\begin{array}{l}\text { Methylprednisolone } \\
\text { acetate }^{\circledast}\end{array}$ & $\begin{array}{l}\text { https://www.sfda.gov.sa/ar/drug/news/ } \\
\text { Pages/drugnews1-12-1433-2.aspx }\end{array}$ & contamination with an accidental fungal lead to meningitis \\
\hline 6 & 12 & Yutopar $^{\oplus}$ & $\begin{array}{l}\text { https://www.sfda.gov.sa/ar/drug/news/ } \\
\text { Pages/drugnews19-05-13a1.aspx }\end{array}$ & Lead to serious side effects such as cardiovascular disease \\
\hline 7 & 13 & Protelos ${ }^{\oplus}$ & $\begin{array}{l}\text { https://www.sfda.gov.sa/ar/drug/news/ } \\
\text { Pages/drognews20-07-2014a1.aspx }\end{array}$ & Lead to serious side effects such as cardiovascular disease \\
\hline 8 & 14 & Omafen ${ }^{\oplus}$ & $\begin{array}{l}\text { https://www.sfda.gov.sa/ar/drug/news/ } \\
\text { pages/drug03-03-2016a1.aspx }\end{array}$ & Contamination of small aluminum foil in the strips \\
\hline 9 & 15 & Loric $^{\circledast}$ & $\begin{array}{l}\text { https://www.sfda.gov.sa/ar/drug/ } \\
\text { circulations/DocLib2/LORIC-300MG- } \\
\text { TABLET.pdf }\end{array}$ & $\begin{array}{l}\text { Non-compliance with manufacturer's specifications-does not } \\
\text { pass the solubility test }\end{array}$ \\
\hline 10 & 16 & Peptac ${ }^{\oplus}$ & $\begin{array}{l}\text { https://www.sfda.gov.sa/ar/drug/ } \\
\text { circulations/DocLib1/PEPTAC-150MG- } \\
\text { TABLET.pdf }\end{array}$ & Non-compliance with manufacturer's specifications \\
\hline 11 & 17 & Triaxone $^{\oplus}$ & $\begin{array}{l}\text { https://www.sfda.gov.sa/ar/drug/ } \\
\text { circulations/DocLib1/(sterile_water).pdf }\end{array}$ & Similar trade name of another product \\
\hline 12 & 18 & Enoxirt ${ }^{\oplus}$ & $\begin{array}{l}\text { https://www.sfda.gov.sa/ar/drug/ } \\
\text { circulations/DocLib1/Lidocaine-HCL.pdf }\end{array}$ & An error in packaging stage \\
\hline \multirow[t]{4}{*}{13} & 19 & Human albunim ${ }^{\oplus}$ & $\begin{array}{l}\text { https://www.sfda.gov.sa/ar/drug/ } \\
\text { circulations/DocLib1/Human-Albumin.pdf }\end{array}$ & Contaminated with ethyleneglycol \\
\hline & 20 & Human albunim ${ }^{\ominus}$ & $\begin{array}{l}\text { https://www.sfda.gov.sa/ar/drug/ } \\
\text { circulations/DocLib1/Human-Albumin.pdf }\end{array}$ & Contaminated with ethyleneglycol \\
\hline & 21 & Human albunim ${ }^{\ominus}$ & $\begin{array}{l}\text { https://www.sfda.gov.sa/ar/drug/ } \\
\text { circulations/DocLib1/Human-Albumin.pdf }\end{array}$ & Contaminated with ethyleneglycol \\
\hline & 22 & Human albunim ${ }^{\ominus}$ & $\begin{array}{l}\text { https://www.sfda.gov.sa/ar/drug/ } \\
\text { circulations/DocLib1/Human-Albumin.pdf }\end{array}$ & Contaminated with ethyleneglycol \\
\hline 14 & 23 & Dextrose ${ }^{\oplus}$ & $\begin{array}{l}\text { https://www.sfda.gov.sa/ar/drug/ } \\
\text { circulations/DocLib1/dextrose.pdf }\end{array}$ & $\begin{array}{l}\text { Non-compliance with manufacturer's specifications-do } \\
\text { pass physical test }\end{array}$ \\
\hline 15 & 24 & Metronidazole ${ }^{\oplus}$ & $\begin{array}{l}\text { https://www.sfda.gov.sa/ar/drug/ } \\
\text { circulations/DocLib1/METRONIDAZOLE- } \\
\text { INJECTION.pdf }\end{array}$ & $\begin{array}{l}\text { Non-compliance with manufacturer's specifications—do } \\
\text { pass physical test }\end{array}$ \\
\hline 16 & 25 & Peptac ${ }^{\oplus}$ & $\begin{array}{l}\text { https://www.sfda.gov.sa/ar/drug/ } \\
\text { circulations/DocLib1/peptac.pdf }\end{array}$ & Non-compliance with manufacturer's specifications \\
\hline
\end{tabular}


Table 1 Drug recalls data (January 2010 - January 2019) (Continued)

\begin{tabular}{|c|c|c|c|c|}
\hline $\begin{array}{l}\text { No. of } \\
\text { recall } \\
\text { letters }\end{array}$ & $\begin{array}{l}\text { No. of } \\
\text { recall } \\
\text { drugs }\end{array}$ & Trade name & Reference of recall (web link) & Reason of recall \\
\hline 17 & 26 & Paracetol ${ }^{\oplus}$ & $\begin{array}{l}\text { https://www.sfda.gov.sa/ar/drug/ } \\
\text { circulations/DocLib1/paracetol.pdf }\end{array}$ & $\begin{array}{l}\text { Non-compliance with manufacturer's specifications- } \\
\text { contaminated with black impurities }\end{array}$ \\
\hline 18 & 27 & Gentacin ${ }^{\circledR}$ & $\begin{array}{l}\text { https://www.sfda.gov.sa/ar/drug/ } \\
\text { circulations/DocLib1/GENTACIN.pdf }\end{array}$ & $\begin{array}{l}\text { Non-compliance with manufacturer's specifications_-broken } \\
\text { container upon storages }\end{array}$ \\
\hline 19 & 28 & Olfen ${ }^{\oplus}$ & $\begin{array}{l}\text { https://www.sfda.gov.sa/ar/drug/ } \\
\text { circulations/DocLib1/olfen1.pdf }\end{array}$ & $\begin{array}{l}\text { Non-compliance with manufacturer's specifications-do not } \\
\text { pass solubility test }\end{array}$ \\
\hline 22 & 29 & $\begin{array}{l}\text { Vaxigrip single } \\
\text { dose }^{\oplus}\end{array}$ & $\begin{array}{l}\text { https://www.sfda.gov.sa/ar/drug/ } \\
\text { circulations/DocLib1/NAXIGRIP.pdf }\end{array}$ & Non-compliance with manufacturer's specifications \\
\hline 20 & 30 & Omacid $^{\circledR}$ & $\begin{array}{l}\text { https://www.sfda.gov.sa/ar/drug/ } \\
\text { circulations/DocLib1/OMACID.pdf }\end{array}$ & $\begin{array}{l}\text { Non-compliance with manufacturer's specifications-do not } \\
\text { pass assay test }\end{array}$ \\
\hline 21 & 31 & Jusprin ${ }^{\oplus}$ & $\begin{array}{l}\text { https://www.sfda.gov.sa/ar/drug/ } \\
\text { circulations/DocLib1/JUSPRIN-81MG.pdf }\end{array}$ & $\begin{array}{l}\text { Non-compliance with manufacturer's specifications-do } \\
\text { pass dissolution test }\end{array}$ \\
\hline \multirow[t]{4}{*}{23} & 32 & Pedovex ${ }^{\oplus}$ & $\begin{array}{l}\text { https://www.sfda.gov.sa/ar/drug/ } \\
\text { circulations/DocLib1/Clopidogrel.pdf }\end{array}$ & $\begin{array}{l}\text { Non-compliance with manufacturer's specifications-do } \\
\text { pass bioequivalence test }\end{array}$ \\
\hline & 33 & Cardlet $^{\oplus}$ & $\begin{array}{l}\text { https://www.sfda.gov.sa/ar/drug/ } \\
\text { circulations/DocLib1/Clopidogrel.pdf }\end{array}$ & $\begin{array}{l}\text { Non-compliance with manufacturer's specifications-do } \\
\text { pass bioequivalence test }\end{array}$ \\
\hline & 34 & Vidapart $^{\oplus}$ & $\begin{array}{l}\text { https://www.sfda.gov.sa/ar/drug/ } \\
\text { circulations/DocLib1/Clopidogrel.pdf }\end{array}$ & $\begin{array}{l}\text { Non-compliance with manufacturer's specifications-do } \\
\text { pass bioequivalence test }\end{array}$ \\
\hline & 35 & Clopex $^{\otimes}$ & $\begin{array}{l}\text { https://www.sfda.gov.sa/ar/drug/ } \\
\text { circulations/DocLib1/Clopidogrel.pdf }\end{array}$ & $\begin{array}{l}\text { Non-compliance with manufacturer's specifications-do } \\
\text { pass bioequivalence test }\end{array}$ \\
\hline 24 & 36 & Clogre $^{\circledast}$ & $\begin{array}{l}\text { https://www.sfda.gov.sa/ar/drug/ } \\
\text { circulations/DocLib1/clogrel.pdf }\end{array}$ & Non-compliance with manufacturer's specifications \\
\hline 25 & 37 & Lynparza ${ }^{\circledast}$ & $\begin{array}{l}\text { https://www.sfda.gov.sa/ar/drug/ } \\
\text { circulations/DocLib1/LYNPARZA\%205 } \\
\text { Omg.pdf }\end{array}$ & $\begin{array}{l}\text { Non-compliance with manufacturer's specifications-do } \\
\text { pass stability test }\end{array}$ \\
\hline 26 & 38 & Thiamine $^{\oplus}$ & $\begin{array}{l}\text { https://www.sfda.gov.sa/ar/drug/ } \\
\text { circulations/DocLib1/Thiamine100Mg.pdf }\end{array}$ & $\begin{array}{l}\text { Do not pass the (friability test) test, which may affect the } \\
\text { safety, efficacy of the preparation }\end{array}$ \\
\hline 27 & 39 & Metaphage ${ }^{\oplus}$ & $\begin{array}{l}\text { https://www.sfda.gov.sa/ar/drug/ } \\
\text { circulations/DocLib1/MetaphageTablet. } \\
\text { PDF }\end{array}$ & $\begin{array}{l}\text { Non-compliance with manufacturer's specifications-do } \\
\text { pass bioequivalence test }\end{array}$ \\
\hline 28 & 40 & Apo-Primidone ${ }^{\oplus}$ & $\begin{array}{l}\text { https://www.sfda.gov.sa/ar/drug/ } \\
\text { circulations/DocLib1/Apo-Primidone25 } \\
\text { OmgTablets.pdf }\end{array}$ & $\begin{array}{l}\text { Non-compliance with manufacturer's specifications- higher } \\
\text { lead content in the formulation }\end{array}$ \\
\hline 29 & 41 & Leukeran ${ }^{\circledast}$ & $\begin{array}{l}\text { https://www.sfda.gov.sa/ar/drug/ } \\
\text { circulations/DocLib1/Leukeran2MG.TAB. } \\
\text { pdf }\end{array}$ & $\begin{array}{l}\text { Non-compliance with manufacturer's specifications-wrong } \\
\text { write for the Arabic name on the container }\end{array}$ \\
\hline 30 & 42 & Claradone $^{\circledast}$ & $\begin{array}{l}\text { https://www.sfda.gov.sa/ar/drug/ } \\
\text { circulations/Documents/CLARADONE- } \\
\text { OINTMENT.pdf }\end{array}$ & Non-compliance with manufacturer's specifications \\
\hline \multirow[t]{7}{*}{31} & 43 & Valista ${ }^{\oplus}$ & $\begin{array}{l}\text { https://www.sfda.gov.sa/ar/drug/ } \\
\text { circulations/DocLib1/2948_001.pdf }\end{array}$ & $\begin{array}{l}\text { Contamination with } \mathrm{N} \text {-nitrosodimethylamine (NDMA), } \\
\text { carcinogen }\end{array}$ \\
\hline & 44 & Valista ${ }^{\oplus}$ & $\begin{array}{l}\text { https://www.sfda.gov.sa/ar/drug/ } \\
\text { circulations/DocLib1/2948_001.pdf }\end{array}$ & $\begin{array}{l}\text { Contamination with } \mathrm{N} \text {-nitrosodimethylamine (NDMA), } \\
\text { carcinogen }\end{array}$ \\
\hline & 45 & Valista ${ }^{\circledast}$ & $\begin{array}{l}\text { https://www.sfda.gov.sa/ar/drug/ } \\
\text { circulations/DocLib1/2948_001.pdf }\end{array}$ & $\begin{array}{l}\text { Contamination with } \mathrm{N} \text {-nitrosodimethylamine (NDMA), } \\
\text { carcinogen }\end{array}$ \\
\hline & 46 & Co-valista ${ }^{\oplus}$ & $\begin{array}{l}\text { https://www.sfda.gov.sa/ar/drug/ } \\
\text { circulations/DocLib1/2948_001.pdf }\end{array}$ & $\begin{array}{l}\text { Contamination with } \mathrm{N} \text {-nitrosodimethylamine (NDMA), } \\
\text { carcinogen }\end{array}$ \\
\hline & 47 & Co-valista ${ }^{\oplus}$ & $\begin{array}{l}\text { https://www.sfda.gov.sa/ar/drug/ } \\
\text { circulations/DocLib1/2948_001.pdf }\end{array}$ & $\begin{array}{l}\text { Contamination with } \mathrm{N} \text {-nitrosodimethylamine (NDMA), } \\
\text { carcinogen }\end{array}$ \\
\hline & 48 & Co-valista $^{\oplus}$ & $\begin{array}{l}\text { https://www.sfda.gov.sa/ar/drug/ } \\
\text { circulations/DocLib1/2948_001.pdf }\end{array}$ & $\begin{array}{l}\text { Contamination with } \mathrm{N} \text {-nitrosodimethylamine (NDMA), } \\
\text { carcinogen }\end{array}$ \\
\hline & 49 & Co-valista ${ }^{\oplus}$ & $\begin{array}{l}\text { https://www.sfda.gov.sa/ar/drug/ } \\
\text { circulations/DocLib1/2948_001.pdf }\end{array}$ & $\begin{array}{l}\text { Contamination with N-Nitrosodimethylamine (NDMA), } \\
\text { carcinogen }\end{array}$ \\
\hline
\end{tabular}


Table 1 Drug recalls data (January 2010 - January 2019) (Continued)

\begin{tabular}{|c|c|c|c|c|}
\hline $\begin{array}{l}\text { No. of } \\
\text { recall } \\
\text { letters }\end{array}$ & $\begin{array}{l}\text { No. of } \\
\text { recall } \\
\text { drugs }\end{array}$ & Trade name & Reference of recall (web link) & Reason of recall \\
\hline & 50 & Co-valista ${ }^{\oplus}$ & $\begin{array}{l}\text { https://www.sfda.gov.sa/ar/drug/ } \\
\text { circulations/DocLib1/2948_001.pdf }\end{array}$ & $\begin{array}{l}\text { Contamination with } \mathrm{N} \text {-nitrosodimethylamine (NDMA), } \\
\text { carcinogen }\end{array}$ \\
\hline & 51 & Diostar ${ }^{\circledast}$ & $\begin{array}{l}\text { https://www.sfda.gov.sa/ar/drug/ } \\
\text { circulations/DocLib1/2948_001.pdf }\end{array}$ & $\begin{array}{l}\text { Contamination with } \mathrm{N} \text {-nitrosodimethylamine (NDMA), } \\
\text { carcinogen }\end{array}$ \\
\hline & 52 & Diostar $^{\circledast}$ & $\begin{array}{l}\text { https://www.sfda.gov.sa/ar/drug/ } \\
\text { circulations/DocLib1/2948_001.pdf }\end{array}$ & $\begin{array}{l}\text { Contamination with } \mathrm{N} \text {-nitrosodimethylamine (NDMA), } \\
\text { carcinogen }\end{array}$ \\
\hline & 53 & Diostar plus ${ }^{\circledast}$ & $\begin{array}{l}\text { https://www.sfda.gov.sa/ar/drug/ } \\
\text { circulations/DocLib1/2948_001.pdf }\end{array}$ & $\begin{array}{l}\text { Contamination with } \mathrm{N} \text {-nitrosodimethylamine (NDMA), } \\
\text { carcinogen }\end{array}$ \\
\hline & 54 & Diostar plus ${ }^{\circledast}$ & $\begin{array}{l}\text { https://www.sfda.gov.sa/ar/drug/ } \\
\text { circulations/DocLib1/2948_001.pdf }\end{array}$ & $\begin{array}{l}\text { Contamination with } \mathrm{N} \text {-nitrosodimethylamine (NDMA), } \\
\text { carcinogen }\end{array}$ \\
\hline & 55 & Diostar plus ${ }^{\circledast}$ & $\begin{array}{l}\text { https://www.sfda.gov.sa/ar/drug/ } \\
\text { circulations/DocLib1/2948_001.pdf }\end{array}$ & $\begin{array}{l}\text { Contamination with } \mathrm{N} \text {-nitrosodimethylamine (NDMA), } \\
\text { carcinogen }\end{array}$ \\
\hline
\end{tabular}

$56 \quad$ Glymide

$57 \quad$ Diatab ${ }^{\oplus}$

33

$58 \quad$ Parazole $^{\oplus}$

34

59 Taxotere ${ }^{\circledast}$

35

$62 \quad$ Narapril $^{\oplus}$

63
Narapril $^{\circledast}$

Julmentin ${ }^{\circledR}$

Julmentin ${ }^{\circledR}$

Angiotec $^{\circledR}$

Angiotec $^{\circledR}$

Angiotec $^{\circledR}$

Lipomax $^{\circledast}$

Lipomax $^{\oplus}$

Lipomax $^{\circledast}$

Dialon $^{\circledR}$

Smooderm ${ }^{\circledR}$

Lobet $^{\circledast}$ https://www.sfda.gov.sa/ar/drug/ circulations/DocLib1/GlymideDiatab.pdf

https://www.sfda.gov.sa/ar/drug/ circulations/DocLib1/GlymideDiatab.pdf

https://www.sfda.gov.sa/ar/drug/ circulations/Documents/PARAZOLE100. pdf

https://www.sfda.gov.sa/ar/drug/ circulations/DocLib1/Taxotere20mg.pdf

https://www.sfda.gov.sa/ar/drug/ circulations/DocLib1/3361_001.pdf

https://www.sfda.gov.sa/ar/drug/ circulations/DocLib1/3361_001.pdf

https://www.sfda.gov.sa/ar/drug/ circulations/DocLib1/3361_001.pdf

https://www.sfda.gov.sa/ar/drug/ circulations/DocLib1/3361_001.pdf

https://www.sfda.gov.sa/ar/drug/ circulations/DocLib1/3361_001.pdf

https://www.sfda.gov.sa/ar/drug/ circulations/DocLib1/3361_001.pdf

https://www.sfda.gov.sa/ar/drug/ circulations/DocLib1/Drug-circ97.pdf

https://www.sfda.gov.sa/ar/drug/ circulations/DocLib1/Drug-circ97.pdf

https://www.sfda.gov.sa/ar/drug/ circulations/DocLib1/Drug-circ97.pdf

https://www.sfda.gov.sa/ar/drug/ circulations/DocLib1/Drug-circ97.pdf

https://www.sfda.gov.sa/ar/drug/ circulations/DocLib1/Drug-circ97.pdf

https://www.sfda.gov.sa/ar/drug/ circulations/DocLib1/Drug-circ97.pdf

https://www.sfda.gov.sa/ar/drug/ circulations/DocLib1/Drug-circ97.pdf

https://www.sfda.gov.sa/ar/drug/ circulations/DocLib1/SmoodermCream. pdf

https://www.sfda.gov.sa/ar/drug/ circulations/DocLib1/lobet.pdf
Non-compliance with manufacturer's specifications-do not pass bioequivalence assay

Non-compliance with manufacturer's specifications-do not pass bioequivalence assay

Non-compliance with manufacturer's specifications-do not pass bioequivalence assay

Non-compliance with manufacturer's specifications-do not pass bioequivalence assay higher drug concentration than stated

Change the source of active ingredient without SFDA approval

Add different source of the active ingredient without SFDA approval

Add different source of the active ingredient without SFDA approval

Add different source of the active ingredient without SFDA approval

Non-compliance with manufacturer's specifications-do not change the expiration date from 3 to 2 years

Non-compliance with manufacturer's specifications-do not change the expiration date from 3 to 2 years

Non-compliance with manufacturer's specifications-do not pass bioequivalence assay

Non-compliance with manufacturer's specifications-do not pass bioequivalence assay

Non-compliance with manufacturer's specifications-do not pass bioequivalence assay

Non-compliance with manufacturer's specifications-do not pass bioequivalence assay

Non-compliance with manufacturer's specifications-do not pass bioequivalence assay

Non-compliance with manufacturer's specifications-do not pass bioequivalence assay

Non-compliance with manufacturer's specifications-do not pass bioequivalence assay

Non-compliance with manufacturer's specifications-do not write batch number, manufacturing, and expiration date

Non-compliance with manufacturer's specificationspresence of impurity in the formulation 
Table 1 Drug recalls data (January 2010 - January 2019) (Continued)

\begin{tabular}{|c|c|c|c|c|}
\hline $\begin{array}{l}\text { No. of } \\
\text { recall } \\
\text { letters }\end{array}$ & $\begin{array}{l}\text { No. of } \\
\text { recall } \\
\text { drugs }\end{array}$ & Trade name & Reference of recall (web link) & Reason of recall \\
\hline 40 & 75 & Ozurdex ${ }^{\oplus}$ & $\begin{array}{l}\text { https://www.sfda.gov.sa/ar/drug/ } \\
\text { circulations/DocLib1/OZURDEX.pdf }\end{array}$ & $\begin{array}{l}\text { Non-compliance with manufacturer's specifications- } \\
\text { presence of impurity in the formulation }\end{array}$ \\
\hline 39 & 76 & BCG-medac ${ }^{\oplus}$ & $\begin{array}{l}\text { https://www.sfda.gov.sa/ar/drug/ } \\
\text { circulations/DocLib1/BCG-medac.pdf }\end{array}$ & $\begin{array}{l}\text { Non-compliance with manufacturer's specifications-errors } \\
\text { in quality control of the catheter tubes }\end{array}$ \\
\hline 41 & 77 & Azimac ${ }^{\oplus}$ & $\begin{array}{l}\text { https://www.sfda.gov.sa/ar/drug/ } \\
\text { circulations/Documents/AZIMAC_Film_ } \\
\text { coated.pdf }\end{array}$ & $\begin{array}{l}\text { Non-compliance with manufacturer's specifications- } \\
\text { presence of impurity in the formulation }\end{array}$ \\
\hline 42 & 78 & Paracetol $^{\oplus}$ & $\begin{array}{l}\text { https://www.sfda.gov.sa/ar/drug/ } \\
\text { circulations/Documents/PARACETOL_10_ } \\
\text { mg.pdf }\end{array}$ & $\begin{array}{l}\text { Non-compliance with manufacturer's specifications- } \\
\text { presence of impurity in the formulation }\end{array}$ \\
\hline 43 & 79 & Cefuzime $^{\oplus}$ & $\begin{array}{l}\text { https://www.sfda.gov.sa/ar/drug/ } \\
\text { circulations/DocLib1/Cefuzime.pdf }\end{array}$ & $\begin{array}{l}\text { Non-compliance with manufacturer's specifications-do not } \\
\text { pass dissolution test }\end{array}$ \\
\hline 44 & 80 & Riazole $^{\circledast}$ & $\begin{array}{l}\text { https://www.sfda.gov.sa/ar/drug/ } \\
\text { circulations/DocLib1/Riazole125mg.pdf }\end{array}$ & Non-compliance with manufacturer's specifications \\
\hline \multirow[t]{2}{*}{45} & 81 & Losanet $^{\oplus}$ & $\begin{array}{l}\text { https://www.sfda.gov.sa/ar/drug/ } \\
\text { circulations/DocLib1/LOSANET50mg-1 } \\
\text { 00mg.pdf }\end{array}$ & $\begin{array}{l}\text { Contaminated with toxic carcinogenic material } \mathrm{N} \text { - } \\
\text { nitrosodiethylamine (NDEA) }\end{array}$ \\
\hline & 82 & Losanet $^{\oplus}$ & $\begin{array}{l}\text { https://www.sfda.gov.sa/ar/drug/ } \\
\text { circulations/DocLib1/LOSANET50mg-1 } \\
\text { 00mg.pdf }\end{array}$ & $\begin{array}{l}\text { Contaminated with toxic carcinogenic material } \mathrm{N} \text { - } \\
\text { nitrosodiethylamine (NDEA) }\end{array}$ \\
\hline 46 & 83 & Jusprin $^{\circledast}$ & $\begin{array}{l}\text { https://www.sfda.gov.sa/ar/drug/ } \\
\text { circulations/DocLib1/Jusprin81mg.pdf }\end{array}$ & Non-compliance with manufacturer's specifications \\
\hline 47 & 84 & Zordy $\left.\right|^{\circledast}$ & $\begin{array}{l}\text { https://www.sfda.gov.sa/ar/drug/news/ } \\
\text { Pages/d23-1-2019a1.aspx }\end{array}$ & $\begin{array}{l}\text { Non-compliance with manufacturer's specifications-do not } \\
\text { pass drug stability test }\end{array}$ \\
\hline
\end{tabular}

to 52 drug recalls in 2018 (61.9\%), increased six-folds by 2018 (Fig. 1).

Considering the therapeutic class of the drugs recalled (Table 2), nineteenth classes accounted for the incidence of the drug recalls. According to classification of medicinal products, the most affected class was the antihypertensive drugs (23 cases, $27.4 \%$ occurrence) followed by the antibiotic drugs (9 cases, 10.71\% occurrence). While the lowest incidence of occurrence (1 case, $1.2 \%$ occurrence) occurred with the drugs classified under anthelmintic, anticonvulsant, antiosteoporotic, and local anesthetic drugs during the period of the study.

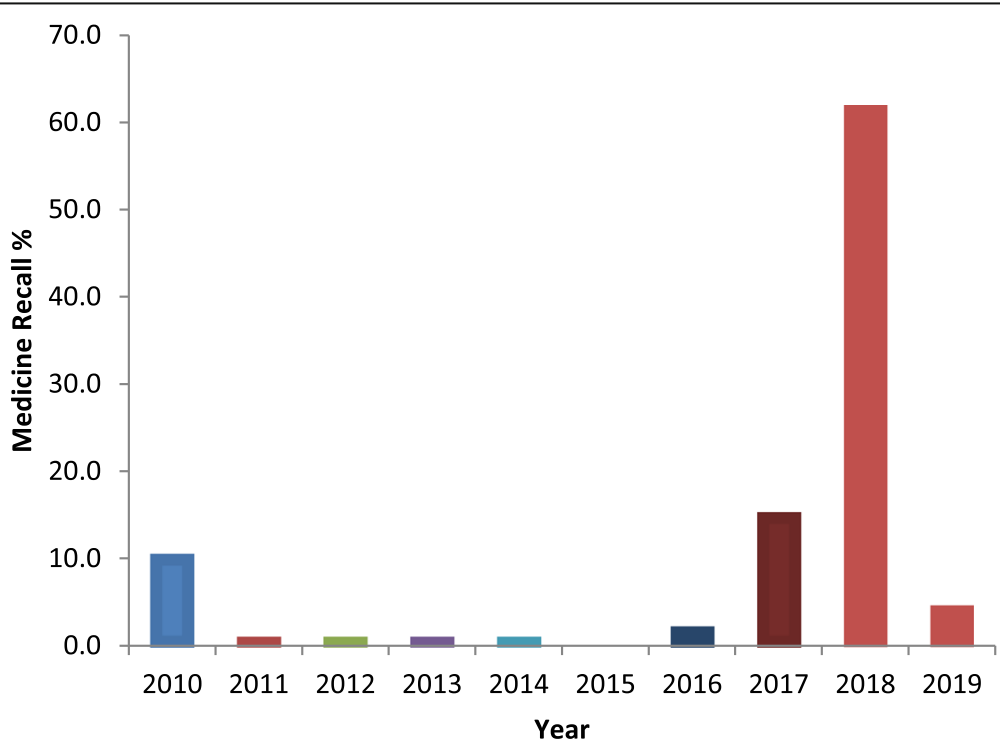

Fig. 1 Percentage of recalled medicines reported by Saudi FDA (January 2010 - January 2019) 
Table 2 Therapeutic class for the drug recall data (2010 - January 2019)

\begin{tabular}{|c|c|c|c|}
\hline No. & No. of occurrence & Class & Occurrence $\%$ \\
\hline 1 & 23 & Antihypertensive & 27.7 \\
\hline 2 & 9 & Antibiotics & 10.8 \\
\hline 3 & 7 & Antidiabetic & 8.4 \\
\hline 4 & 6 & Non-steroidal anti-inflammatory-analgesic & 7.2 \\
\hline 5 & 5 & Antiplatelet & 6.1 \\
\hline 6 & 5 & Bronchodilator-long acting beta agonists & 6.1 \\
\hline 7 & 4 & Plasma volume expander & 4.8 \\
\hline 8 & 3 & Statins_-anticholesterol & 3.5 \\
\hline 9 & 3 & Anticancer & 3.5 \\
\hline 10 & 3 & Antacid & 3.5 \\
\hline 11 & 3 & Corticosteroids & 3.5 \\
\hline 12 & 3 & Vitamin & 3.5 \\
\hline 13 & 2 & Antimicrobial and antiseptic oral rinse & 2.4 \\
\hline 14 & 2 & Vaccine & 2.4 \\
\hline 15 & 2 & Antiobesity & 2.4 \\
\hline 16 & 1 & Anthelmintic & 1.2 \\
\hline 17 & 1 & Anticonvulsant & 1.2 \\
\hline 18 & 1 & Antiosteoporotic & 1.2 \\
\hline 19 & 1 & Local anesthetic & 1.2 \\
\hline total & 84 & 19-therapeutic class & 100 \\
\hline
\end{tabular}

Moreover, with respect to the pharmaceutical dosage forms, the majority of the 84 recall pharmaceutical dosage forms were for tablet formulations (58\%) followed by parenteral dosage forms (25\%) as shown in Fig. 2.

Drug recalls were mainly due to one of the following reasons (Table 3): defective medicinal products $78.06 \%$, adverse reaction $12.05 \%$, or falsified medicinal products $1.2 \%$. The most important cause was a defective medicinal product which was due to one of the following reasons: contamination 32.5\%, non-compliance with manufacturer's specification $21.43 \%$, failure to demonstrate bioequivalence $19.28 \%$, packaging defect $9.64 \%$, and problems related to the active ingredients $4.81 \%$.

The most important problem, with defective medicinal products, in which 27 drugs affected out of 84 (32.14 \%) was due to contamination represented by $N$-nitrosodimethylamine (NDMA, carcinogen), ethylglycol, nonspecified impurities, $\mathrm{N}$-nitrosodiethylamine (NDEA, carcinogen), aluminum foil, fungal contamination, black impurities, or high led content accomplished by $15.66 \%$, $4.82 \%, 4.82 \%, 2.41 \%, 1.2 \%, 1.2 \%, 1.2 \%$, and $1.2 \%$, respectively. The second important reason was the noncompliance with manufacturer's specifications (20.48\%) followed by failure to demonstrate bioequivalence (19.28\%).

The study recognized 33 manufacturers for 84 medicinal products which were recalled by the SFDA. Manufacturers for recalled medicinal products are listed in
Fig. 3. Two manufacturers were found the most commonly denoted for drug recalls. Together, they produced 29 drugs, accounting for $34.52 \%$ of all recalled drugs.

The most frequent dosage forms reported for recall were the tablet formulations accounting for $58 \%$ of incidents.

\section{Discussion}

In general, the SFDA performance has developed since it was started in 2009 [16, 18-21]. The launch and improvement of the SFDA's website as well as the inclusion of an incorporated site for pharmacovigilance created an important communication tool with health care professionals and the public. The pharmacovigilance electronic system is an online automatic reporting service for adverse reactions and defective medicinal products to enhance the reporting for health care professionals and the public $[16,18-21]$. It is now possible to report medicinal products that could be defective online or over the phone if the healthcare professional or public considers that the defect in a medicinal product may produce serious adverse reactions to public health $[16,18-21]$.

The SFDA has launched guidelines to healthcare professionals describing methods for reporting medicinal products that could be defective. These guidelines illustrate the part that healthcare professionals perform in the initial evaluation of the medicinal products that 


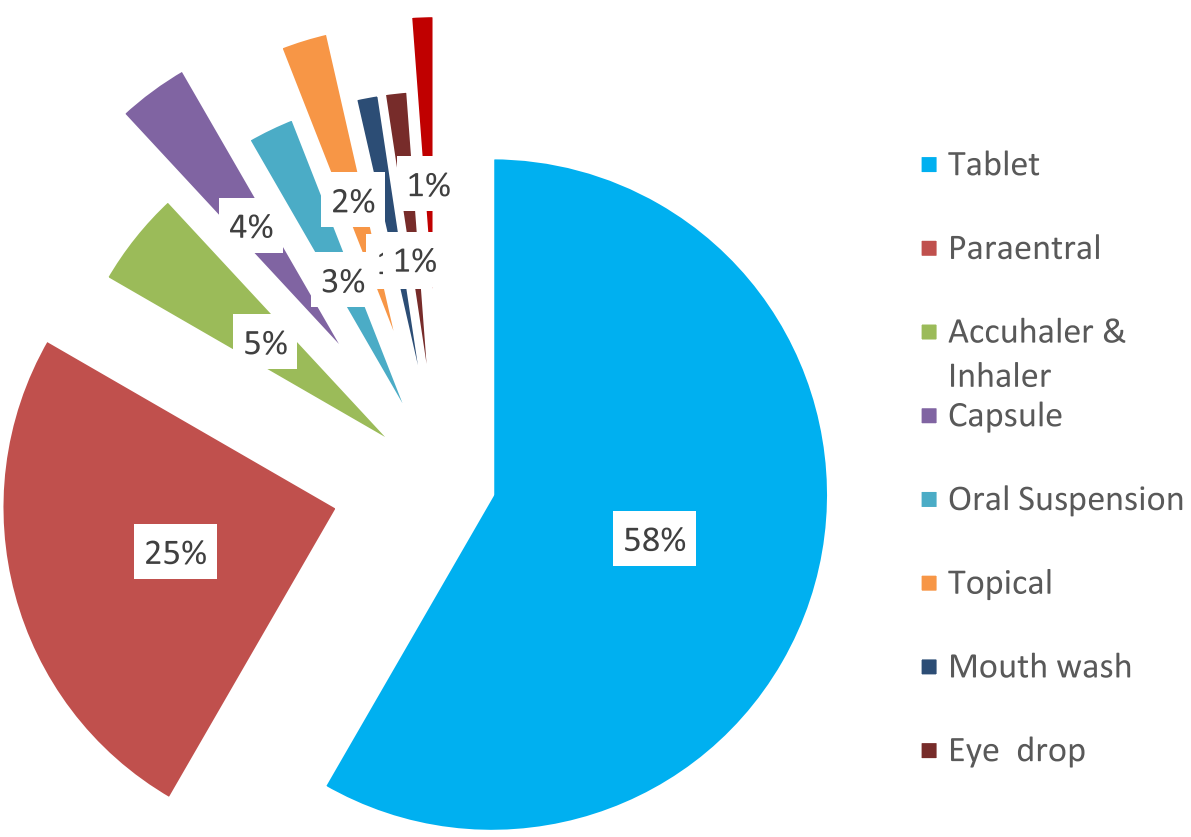

Fig. 2 Types of dosage forms of recalled medicines reported by Saudi FDA

could be defective. They also demonstrate consequent evaluations that can be performed by the SFDA before it reports a drug alert. Moreover, the ease of access through the SFDA's website to these guidelines may enhance healthcare professionals' understanding about how to manage the medicinal products that could be defective [24].

This is the first review that studies the problem of medicinal product recalls in Saudi Arabia by assessing the drug alerts posted on SFDA website.

There were few publications about drug recalled in different countries and categories, such as the retrospective analysis by Freeman et al. [10] that results in a total of 21,120 products which were recalled during the 30 -month study period and recalls of cosmetic and personal care products from 2002 to 2016 [10]. Almuzaini et al. [26] demonstrate a total of 653 defective medicines in Canada during their study from 2005 to 2013.
Between 2002 and 2016, 806 million medical devices were recalled [16].

Our investigations of medicinal product recalls from Jan 2010 to Jan 2019 have shown that the rate of recalled medicinal products increased; however, there is inadequate information to prove this. Also, the main cause behind the increased rate of recalled medicinal products is unknown. It could be due to new regulations and guidelines by the SFDA that have been contributed to easier detection of medicinal product defects, or it could be due to an increased rate of manufacture of defective medicinal products.

This is the first review that discusses the number of substandard and falsified medicines in Saudi Arabia by evaluating the reasons for drug recalls, trade names, and therapeutic classes posted on Saudi FDA website which are not arranged; they posted according to the SFDA recall letter days.

Table 3 Reasons for the drug recall data (2010 - January 2019)

\begin{tabular}{llll}
\hline Reason for recall & Number of medicinal product & Percent \% \\
\hline 1 & Quality defects & 73 & 78.06 \\
1. Contamination & 27 & 32.53 \\
2. Non-compliance with manufacturer's specifications & 18 & 21.43 \\
3. Failure to demonstrate bioequivalence & 16 & 19.28 \\
4. Problems related to packaging & 8 & 9.64 \\
& 5. Problems related to the active ingredients & 4.81 \\
& Adverse reaction & 4 & 12.05 \\
& Falsified medicine & 10 & 1.20 \\
\hline
\end{tabular}




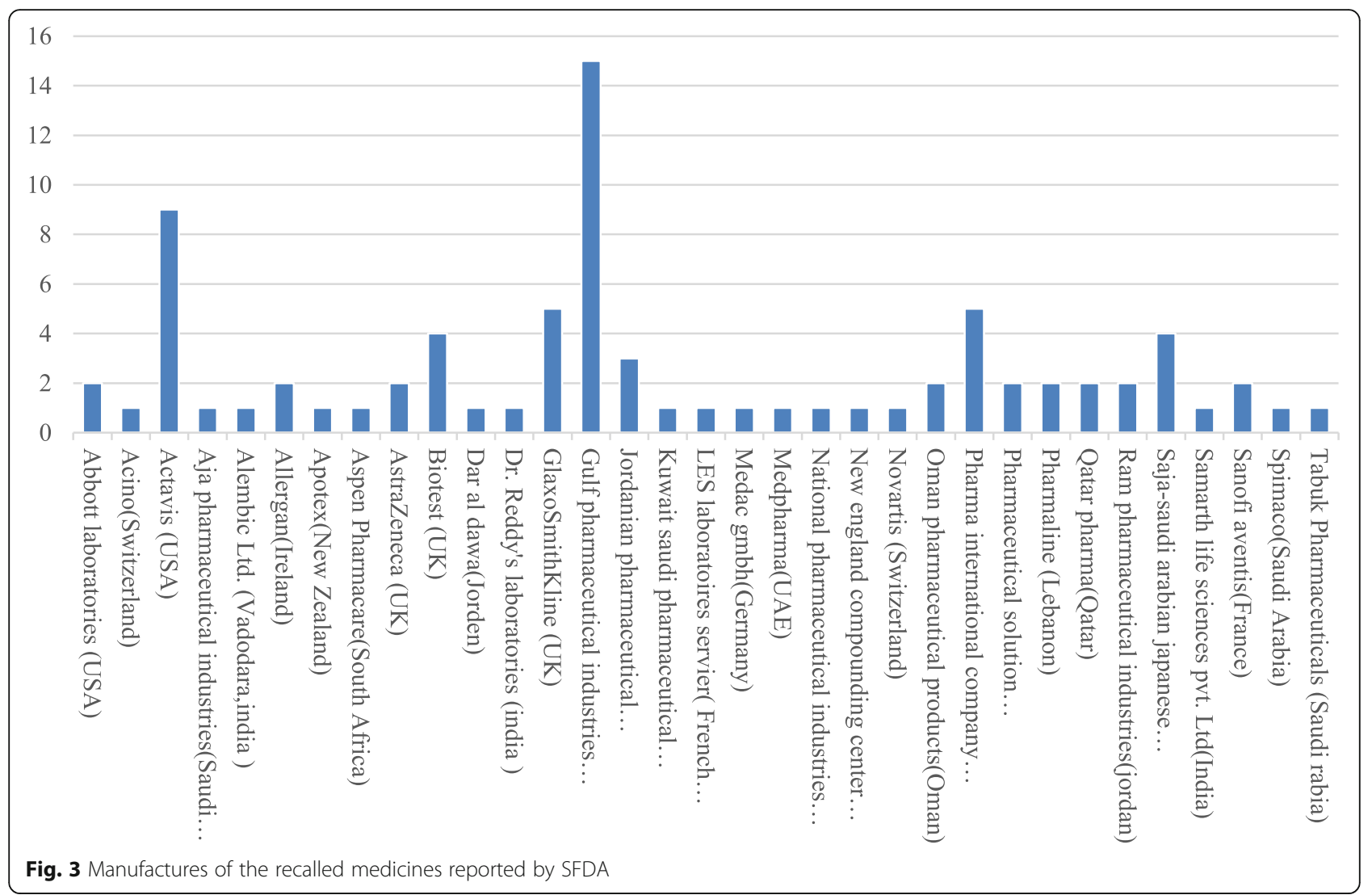

During the period of the study (2009-2019), the study over ten consecutive years has shown the recall for substandard medicines is an increasing trend, and the drug recalls increased six-folds by 2018 . The most affected therapeutic class was the antihypertensive and antibiotic drugs $(27.4 \%$ and $10.71 \%$, respectively.

Not surprisingly, over half numbers of recalls $(58 \%)$ for tablet formulations (58\%) followed by parenteral dosage forms (25\%). Similarly, Almuzaini et al. found that the most frequent type of formulations reported to be defective were tablets $[26,27]$. The sterility precaution during the manufacturing process of tablet formulations is less than that required during parenteral formulations. However, serious adverse reactions can results as reported in January 2012 in Pakistan. Isosorbide mononitrate tablets were contaminated with large doses of pyrimethamine (an antimalarial drug) which lead to the death of 151 patients and 664 cases of pyrimethamine toxicity [11].

Medicinal product contamination is considered as a major problem. The Food and Drug Administration (FDA) recalled all unused corticosteroid medication vials for injection in October 2012. The reason was vial contamination with various bacterial/fungal strains. The use of contaminated vials leads to 64 deaths and 751 incidents [26]. Contamination was the first significant reason for drug recalls followed by non-compliance with manufacturer's specification, then failure to demonstrate bioequivalence $(32.5,21.43,19.28 \%)$, respectively. Similarly, Hall et al. carried out a retrospective study evaluating the relationship between recall companies and medicines recalled by FDA from June 20, 2012, to December 31, 2014. They found that the most common reasons for drug recall were contamination (50.1\%) [10]. Also, Almuzaini et al. evaluated drug alerts in the UK (2001-2011), they found that contaminations are the most common reasons for drug recall (27\%) [25].

Thirty-three manufacturers were recorded for the 84 medicinal products which were recalled by the SFDA. Two manufacturers were found the most commonly denoted for drug recalls $34.52 \%$.

\section{Conclusion}

Saudi Arabia is one of the countries which affecting by the problem of the substandard medicinal products. An investigation of SFDA drug recalls (Jan 2010 and Jan 2019) revealed that contaminations of the drug products were the most cause of drug recalls, and tablet formulations considered the most affecting dosage forms. The most affected therapeutic class was the antihypertensive drugs followed by the antibiotics drugs. Both healthcare professionals and consumers should be stayed informed actively about drug safety and drug recalls. 


\section{Supplementary information}

Supplementary information accompanies this paper at https://doi.org/10. 1186/s43094-020-00112-3.

Additional file 1. Tha Excell collection data from January 2010 January 2019 taken from the SFDA website.

\section{Abbreviations}

ATC: Anatomical Therapeutic Chemical Classification System; NDMA: NNitrosodimethylamine; NDEA: Ethylglycol, non-specified impurities; SA: Saudi Arabia; SFDA: Saudi Food and Drug Administration

\section{Acknowledgements}

This research project was supported by a grant from the "Research Center of the Female Scientific and Medical Colleges," Deanship of Scientific Research, King Saud University.

\section{Authors' contributions}

BA conceptualized the whole idea, worked as the project administration, supervised the whole other members, analyzed the data, wrote the original draft, and reviewed and edited the manuscript. IA contributed to the analysis of the data, writing of the original draft, and review and editing of the manuscript. AA, SA, and RA are bachelor students, who collected the data from the web site, arranged it in Excel sheet, and double-checked the data. All authors have read and approved the manuscript and ensure that this is the case.

\section{Funding}

No funding was received.

\section{Availability of data and materials}

All data and materials are available upon request.

\section{Ethics approval and consent to participate}

Not applicable

\section{Consent for publication}

Not applicable

\section{Competing interests}

The authors declare that they have no competing interests.

Received: 20 January 2020 Accepted: 15 September 2020

Published online: 20 November 2020

\section{References}

1. Nagaich U, Sadhna D (2015) Drug recall: an incubus for pharmaceutical companies and most serious drug recall of history. Int J Pharmaceutical Invest 5:13-19

2. Mackey TK, Liang BA (2011) The global counterfeit drug trade: patient safety and public health risks. J Pharm Sci 100:4571-4579

3. Sato D (2014) Counterfeit medicines--Japan and the world. Yakugaku Zasshi 134:213-222

4. Ahearn DG, Stulting RD (2018) Moulds associated with contaminated ocular and injectable drugs: FDA recalls, epidemiology considerations, drug shortages, and aseptic processing. Med Mycol 56:389-394

5. Shakoor O, Taylor R, Behrens R (1997) Assessment of the incidence of substandard drugs in developing countries. Tropical Med Int Health 2:839845

6. Nsimba SE (2009) Problems associated with substandard and counterfeit drugs in developing countries: a review article on global implications of counterfeit drugs in the era of anti-retroviral (ARVS) drugs in a free market economy. East Afr J Public Health 11:311-316

7. Hanson C, Carcillo R, Maffioli E, Aneja R, Pudjiadi A, Horvat C (2020) Global distribuation of counterfeit antimicrobials reflects diparty in pediatric sepsis mortality. Crit Care Med 48:754

8. Lade S, Burle S, Kosalge S, Telrandhe U (2020) Multiple anticounterfeit technologies to compact counterfeiting in pharma industery: an overview. Int J Pharm Res Technol 10:15-24
9. Newton PN, Green MD, Fernández FM (2010) Impact of poor-quality medicines in the 'developing'world. Trends Pharmacol Sci 31:99-101

10. Hall K, Stewart T, Chang J, Freeman M (2016) Characteristics of FDA drug recalls: a 30-month analysis. Am J Health Syst Pharm 73:235-240

11. Imran M, Shafi H, Mahmood Z, Sarwar M, Usman H, Tahir M et al (2016) Fatal intoxications due to administration of isosorbide tablets contaminated with pyrimethamine. J Forensic Sci 1:1382-1385

12. CDC (2015). Centers for Disease Control and Prevention multistate outbreak of fungal meningitis and other infections. https://www.cdc.gov/hai/ outbreaks/meningitis.html.

13. Gershman M, Kennedy D, Noble-Wang J (2008) Multistate outbreak of Pseudomonas fluorescens bloodstream infection after exposure to contaminated heparinized saline flush prepared by a compounding pharmacy. Clin Infect Dis 47:1372-1379

14. Organization WH (2017) Essential medicines and health products (2006). World Health Organization. http://158.232.12.119/medicines/publications/ annual-reports/WHO_EMP_Report_2016_Online.pdf

15. Liang BA (2006) Fade to black: importation and counterfeit drugs. Am J Law Med 32:279-323

16. Ghobadi CW, Janetos TM, Tsai S, Beaumont JL, Welty L, Walter JR et al (2019) Approval-adjusted recall rates of high-risk medical devices from 2002-2016 across Food and Drug Administration device categories. Iss L Med 34:77

17. Janetos TM, Akintilo L, Xu S (2019) Overview of high-risk Food and Drug Administration recalls for cosmetics and personal care products from 2002 to 2016. J Cosmet Dermatol 18:1361-1365

18. SFDA (2017). https://www.sfda.gov.sa/en/about/Pages/overview.aspx.

19. SFDA (2017). https://www.sfda.gov.sa/ar/about/Pages/AnnualReport.aspx..

20. SFDA (2018). https://www.sfda.gov.sa/en/drug/drug_reg/Regulations/SFDAGMP2018.pdf

21. SFDA (2014). https://www.sfda.gov.sa/ar/about/Pages/AnnualReport.aspx.

22. SFDA (2015). https://www.sfda.gov.sa/ar/about/Pages/AnnualReport.aspx

23. SFDA (2016). https://www.sfda.gov.sa/ar/about/Pages/AnnualReport.aspx

24. SFDA (2018). https://www.sfda.gov.sa/ar/about/Pages/AnnualReport.aspx.

25. World Health Organization 2019.

26. Almuzaini T, Sammons H, Choonara I (2014) Quality of medicines in Canada: a retrospective review of risk communication documents (2005-2013). BMJ Open 4:e006088

27. Almuzaini T, Sammons H, Choonara I (2013) Substandard and falsified medicines in the UK: a retrospective review of drug alerts (2001-2011). BMJ Open 3:e002924. https://doi.org/10.1136/bmjopen-2013

\section{Publisher's Note}

Springer Nature remains neutral with regard to jurisdictional claims in published maps and institutional affiliations.

\section{Submit your manuscript to a SpringerOpen ${ }^{\bullet}$ journal and benefit from:}

- Convenient online submission

- Rigorous peer review

- Open access: articles freely available online

- High visibility within the field

- Retaining the copyright to your article

Submit your next manuscript at $>$ springeropen.com 